\title{
Visualization and Analysis of 3D Microscopic Images
}

\author{
Fuhui Long, Jianlong Zhou, Hanchuan Peng* \\ Janelia Farm Research Campus, Howard Hughes Medical Institute, Ashburn, Virginia, United States of America
}

Abstract: In a wide range of biological studies, it is highly desirable to visualize and analyze threedimensional (3D) microscopic images. In this primer, we first introduce several major methods for visualizing typical 3D images and related multi-scale, multi-timepoint, multi-color data sets. Then, we discuss three key categories of image analysis tasks, namely segmentation, registration, and annotation. We demonstrate how to pipeline these visualization and analysis modules using examples of profiling the single-cell geneexpression of $C$. elegans and constructing a map of stereotyped neurite tracts in a fruit fly brain.

\section{Introduction}

Multidimensional microscopic image data sets (Figure 1) are widely used in modern biology studies, especially in screening various phenotypic data. Analyzing microscopic data is highly useful and fruitful, such as observing the dynamics of microtubule spindles during mitosis [1], profiling gene expression of cells [24], and reconstructing the three-dimensional (3D) morphology of neurons [5-7]. Image visualization also enables effective development of high-content highthroughput bioimage informatics techniques [8] to extract biologically meaningful knowledge from microscopic images. It is also critical for visualizing raw images and respectively processed results (in terms of surface objects).

In this primer, we briefly introduce the basic concepts and methods of 3D microscopic image visualization and analysis, which are the two core components for a number of bioimage informatics applications. We emphasize fluorescent microscopic images as examples, and occasionally also mention other types of image data in our discussion. On the other hand, the essential visualization and analysis methods introduced here can be applied to a wide range of data, including many of those not explicitly discussed. Due to the length limitations of this educational note, here we do not intend to comprehensively survey software tools or biological applications, which can be found in a few previous reviews [8-11].

\section{Visualization of 3D Microscopic Images}

Visualizing 3D microscopic images helps better understand the data. It also helps determine appropriate analysis methods or parameters. In addition, visualizing analysis results on top of, or side-by-side with, the input image(s) is critical for checking the meaningfulness of an analysis and making necessary corrections ("proof-editing" [12]).

Two-dimensional (2D) cross-sectional display (Table 1) of a 3D image stack is still the most prevailing method for biologists to observe 3D data sets, probably due to its simplicity. ImageJ [13] (a newer variant bears the name Fiji), a popular tool to visualize and analyze microscopic images, uses mainly the z-section display to visualize 3D images, although various additional ImageJ modules or plugins were also developed to render $3 \mathrm{D}$ views. Tri-view display (Table 1) is a natural extension of the $\mathrm{z}$-slice display of 3D data, displaying all $\mathrm{XY}, \mathrm{XZ}$, and ZY cross-sectional planes at the same time. Cutting through the volumetric data from an arbitrary angle and displaying the 2D image data on this cutting plane is also useful. These features have been incorporated in other scientific visualization software packages (e.g., Vaa3D (previously known as V3D [14]) or GoFigure [15]). Electronic microscopic (EM) images typically have a large cross-sectional size in the $\mathrm{XY}$ plane. It is particularly convenient to view EM images using $2 \mathrm{D}$ or tri-view display methods, such as in the ImageJ-based software TrakEM [16].
However, cross-sectional views are not able to visualize the $3 \mathrm{D}$ information of volumetric images. Visualizing the complete $3 \mathrm{D}$ information in a volumetric image requires seeing (a) all individual image voxels' (pixels) intensity, and (b) the 3D spatial adjacency information of all voxels. However, since normally a rendered image is a $2 \mathrm{D}$ projection to a computer screen and our retina, it is hard to meet both requirements at the same time. Tiling all image voxels on a single 2D plane will not appropriately display the 3D spatial adjacency information. On the other hand, in a 3D volumetric rendering, while the spatial adjacency relationship is retained, not all image voxels' intensity is visible, as voxels near the viewer will occlude far-away voxels. Therefore, selectively discarding the non-important voxel intensity information is the central trick used in 3D volumetric image visualization.

3D image visualization calls for depthblended views from any angle. Maximal (or minimal) intensity projection (MIP or $\mathrm{mIP}$ ) and alpha-value blended views (Table 1) are two main types of methods to display $3 \mathrm{D}$ data. MIP is mainly used to visualize high-intensity structures within volumetric data. This is the typical situation for most fluorescent microscopic (FM) images, e.g., GFP-labeled neuron structures. Usually, MIP contains no shading information; depth and occlusion information are not visible. Structures with higher intensity value lying behind a lower valued object appear to be in front of it. Thus, MIP may not accurately display the actual 3D relationships of structures. While alphablended views can display the depth information more meaningfully, the most common solution is to animate or interactively change the viewpoint while viewing

Citation: Long F, Zhou J, Peng H (2012) Visualization and Analysis of 3D Microscopic Images. PLoS Comput Biol 8(6): e1002519. doi:10.1371/journal.pcbi.1002519

Editor: Fran Lewitter, Whitehead Institute, United States of America

Published June 14, 2012

Copyright: (c) 2012 Long et al. This is an open-access article distributed under the terms of the Creative Commons Attribution License, which permits unrestricted use, distribution, and reproduction in any medium, provided the original author and source are credited.

Funding: The authors are employed by the Howard Hughes Medical Institute. No specific funding was received for this article.

Competing Interests: The authors have declared that no competing interests exist.

* E-mail: pengh@janelia.hhmi.org 

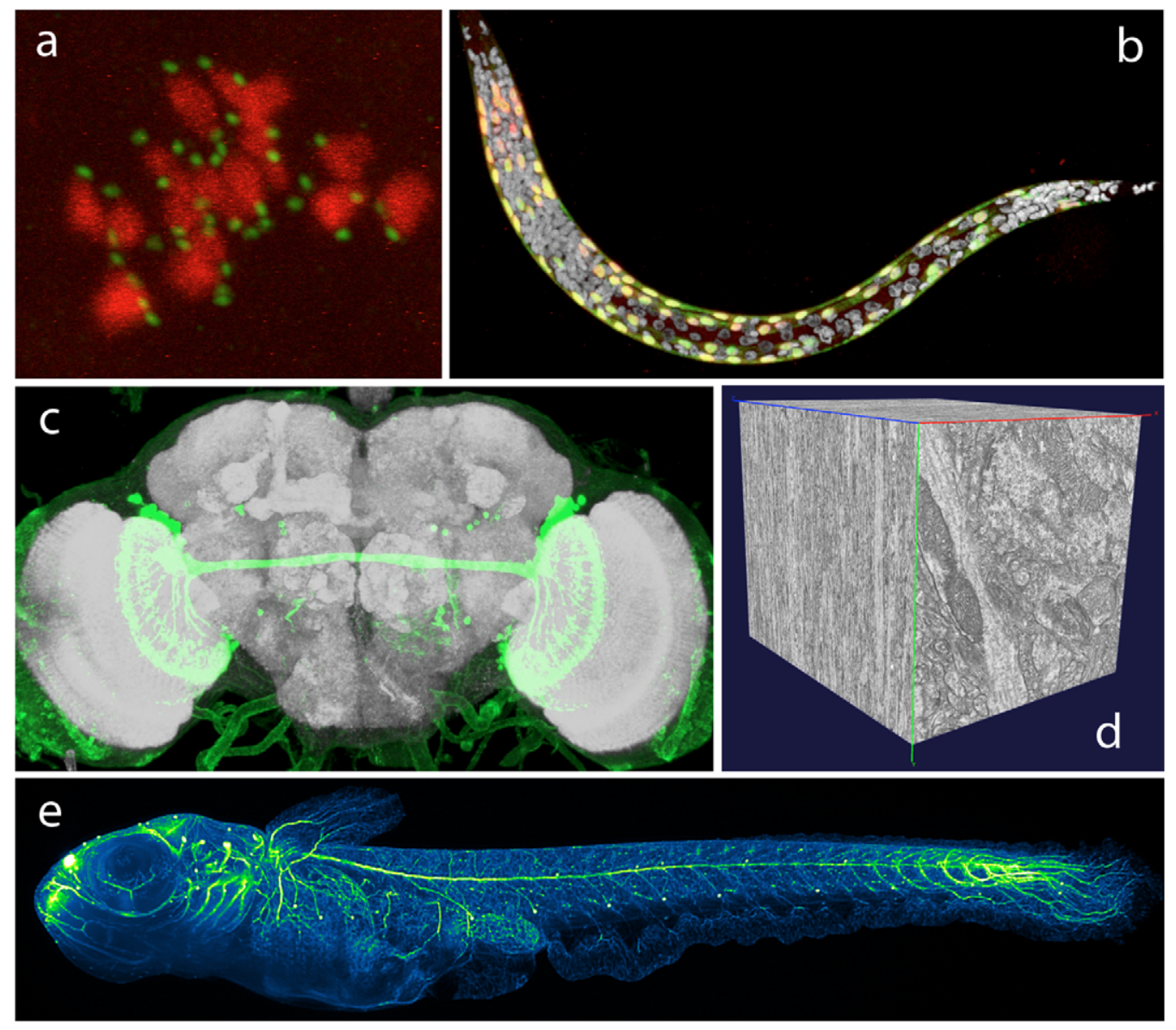

Figure 1. Examples of 3D microscopic images. (a) A confocal image of kinetochores (EGFP labeled) and chromosomes (histone-mCherry labeled) used in studying the first meiotic division in mouse oocytes [17]. (b) A confocal image of the first larval stage of C. elegans [18]. Gray: DAPI labeled nuclei; yellow: myo3:EGFP. (c) A confocal image of an adult fruit fly brain [19]. Gray: NC82 labeled neuropil; green: ato-GAL4 (courtesy of Julie Simpson). (d) A serial section electron microscopic image of mouse visual cortex [20]. (e) A digital scanned laser light sheet fluorescence microscopic image of a Medaka juvenile [21]. Green: acetylated tubulin immuno-staining of the developing brain and spinal cord. doi:10.1371/journal.pcbi.1002519.g001

using MIP (or even alpha-blended views). Therefore, a real-time 3D renderer for large datasets is highly desirable. This often needs both good hardware (i.e., highthroughput graphics card with large memory) and optimized software (e.g., to optimize the OpenGL-based graphics rendering). Vaa3D (http://vaa3d.org) meets this requirement and has been used in

Table 1. Often-used visualization methods for multi-dimensional microscopic image data.

\begin{tabular}{|c|c|c|c|c|c|}
\hline Visualization mode & Dimension & Often Applied to & Pros & Cons & Example Figure(s) \\
\hline Cross-sectional view(s) & 2D & FM/WM/EM & Fast & Not 3D & - \\
\hline Tri-view & 2D & FM/EM & Fast & Partial 3D & 3a \\
\hline Maximum intensity projection (MIP) & 3D & FM & 3D & Hardware-limited (HL) & $1 a, 1 b, 1 c, 1 e, 2 b$ \\
\hline Alpha-value blending & 3D & FM/EM & Surface-display effect & $\mathrm{HL}$ & $1 d, 2 a$ \\
\hline $\begin{array}{l}\text { Multi-channel/multi-color } \\
3 D(M C-3 D)\end{array}$ & 4D & FM & 3D & $\begin{array}{l}\text { Need color-blending } \\
\text { (CB), HL }\end{array}$ & $1 a, 1 b, 1 c, 1 e, 2 b$ \\
\hline Multi-time-point MC 3D (MT-MC-3D) & 5D & FM & 3D & $\mathrm{CB}, \mathrm{HL}$ & $2 \mathrm{~b}$ \\
\hline $\begin{array}{l}\text { Multi-scale MT-MC 3D } \\
\text { (MS-MT-MC-3D) }\end{array}$ & $6 \mathrm{D}$ & FM & $\begin{array}{l}\text { Hardware-friendly, } \\
\text { 3D }\end{array}$ & $\begin{array}{l}\text { Need 3D interaction } \\
\text { of image content }\end{array}$ & $2 a$ \\
\hline $\begin{array}{l}3 D \text { surface-object and } \\
\text { image rendering }\end{array}$ & $\begin{array}{l}\text { Heterogeneous } \\
3 \mathrm{D} / 4 \mathrm{D} / 5 \mathrm{D} / 6 \mathrm{D}\end{array}$ & FM/EM & $\begin{array}{l}\text { Allow proof- } \\
\text { reading/editing }\end{array}$ & $\mathrm{HL}$ & $2 a, 4 b$ \\
\hline
\end{tabular}

CB, color-blending; EM, electron microscopic images; FM, fluorescent microscopic images (often laser-scanning-microscopic images); HL, hardware-limited; WM, widefield light microscopic images.

doi:10.1371/journal.pcbi.1002519.t001 
recent large-scale 3D image visualization applications, e.g., the Janelia Fly WorkStation that currently screens more than 50 terabytes of fruit fly brain images (private communication with the Janelia FlyLight project).

In many cases, each voxel in a $3 \mathrm{D}$ microscopic image could have multiple color components that correspond to various features of the biological entities (e.g., different fluorephores with different wavelengths in fluorescent imaging). Visualizing multi-channel (MG) 3D image stacks (thus four-dimension [4D], see Table 1) requires blending the data in different channels to the RGB space for rendering. When the number of channels, $\mathcal{N}$, is not larger than 3, a simple mapping, e.g., channel 1 to Red or Magenta, channel 2 to Green, and channel 3 to Blue, is often used. When $\mathcal{N}>3$, e.g., in the cases of dozens of co-localized antibody-probes, or thousands of 3D registered image stacks organized as different channels [19], a spreadsheet-based color-blending manager (e.g., the one provided in Vaa3D) will be critical for effective visualization.
Live imaging experiments produce multi-time-point (MT) multi-color 3D image series (thus five-dimension [5D], see Table 1). In addition, when an image is large (e.g., 20 Gbytes/image), it is usually impractical and also unnecessary to load all image voxels in the computer memory and graphics card to visualize. Thus, there is a need to visualize an image dataset at multiple scales. The MT-MC-3D data sets, and multi-scale (MS) rendering (thus six-dimensional visualization [6D], see Table 1), impose significant challenges to current visualization hardware and software, due to the limited bandwidth between hard drives, computer memory, and graphics card. When the entire image series could be loaded in computer memory, Vaa3D could be used to produce real-time 5D or 6D rendering (Figure 2). Yet, in general they are unsolved problems for terabyte-sized image data sets.

Surface-object rendering (Table 1) is a powerful way to visualize image analysis results (e.g., image segmentation) and provides ways for quantitative measurement or editing. Isosurface-based mesh- extraction and rendering has also been used in 3D biomedical image visualization. However, commonly used algorithms, e.g., marching cubes [22], are computationally expensive. In addition, isosurfaces can hardly capture the internal structures in a 3D image.

Interactive visualization techniques are important for microscopic image analysis. Through interactions, users can collect much more information of the multidimensional data than passively observing the $3 \mathrm{D}$ rendered data. Interacting with $3 \mathrm{D}$ rendered surface-objects is straightforward. It is more difficult to directly interact with $3 \mathrm{D}$ rendered volumetric data to define interesting 3D locations, 3D curves, and other objects. The concept of 3D-WYSIWYG (what you see is what you get) was recently proposed in the Vaa3D system to define an unambiguous 3D location (point) using one computer mouse click, or define a unique 3D curve using one mouse stroke on the 2D computer screen. This approach has been demonstrated to boost both the reconstruction speed and accu-

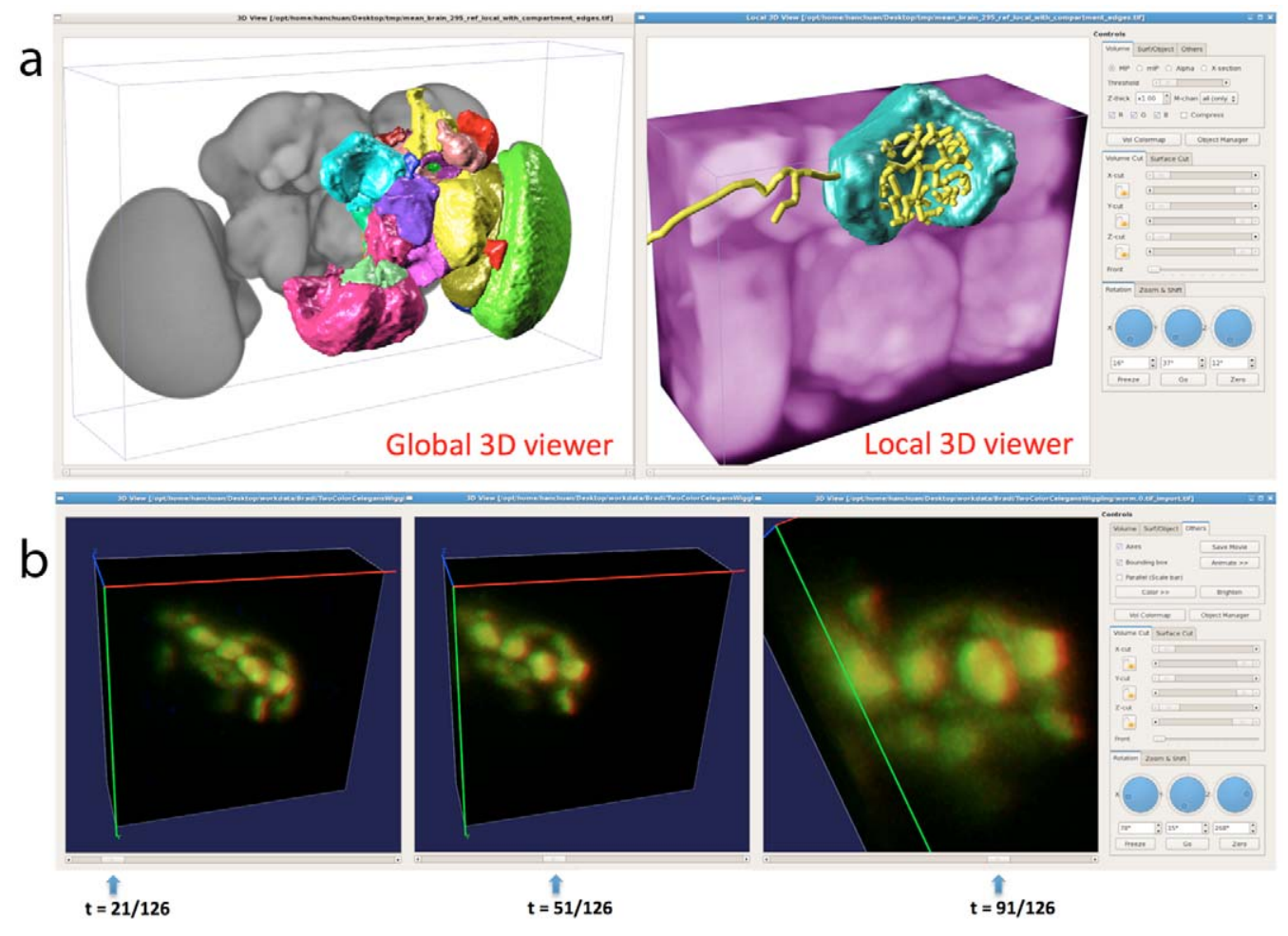

Figure 2. Vaa3D visualization of 4D and 5D microscopic images, as well as associated 3D surface objects, of different model animals. (a) The hierarchical (multi-scale) 3D visualization of a fluorescent confocal image of fruit fly (Drosophila melanogaster) brain using both global and local 3D viewers. In the global viewer, different brain compartments rendered using surface meshes (in different colors) are overlaid on top of the 3D volume of a fruit fly brain. When an image is very large, the global viewer can serve for navigation purpose. A user can quickly define any 3D local region of interest and display it in a local 3D viewer using full resolution. In this example, the brain voxels can be rendered in a different color from the global viewer, while the user can optionally display other surface objects, such as the single 3D-reconstructed neuron (yellow). (b) 5D visualization of a series of multi-color 3D image stacks of $C$. elegans (courtesy of Rex Kerr). Different 3D viewing angles can be adjusted in real-time in Vaa3D, with which the user can freely change the displayed time point (bottom).

doi:10.1371/journal.pcbi.1002519.g002 
racy of 3D neuron morphology [12,14]. In the long run, integrating these 3D interaction techniques in immersive visualization of very large data, possibly also equipped with other virtual reality techniques and a very large display wall, may demonstrate its power in detecting interesting patterns or associations in very large data sets.

In practice, 3D visualization of multidimensional image data may involve many other considerations. For instance, in both 3D tomographic EM imaging and laser scanning microscopy, anisotropy is an often seen property of the data. Software tools (e.g., Vaa3D) can reslice the data in the 3D rendering based on the relative pixel size in three dimensions, thus providing a more realistic display of the data. In Vaa3D, this auto-slicing function is combined with some image analysis functions (e.g., fibrous structure tracing) discussed below to generate various 3D reconstructions of the image objects. In addition, data filtering techniques (e.g., non-linear anisotropic diffusion, recursive median filtering, bilateral filtering, etc.) have been provided in many software tools (e.g., ImageJ). Integrating all these tools together could lead to more interesting insight in the data (see the last section on "pipelining").

\section{Analysis of 3D Microscopic Images}

The overarching goal of microscopic image analysis is to quantitatively measure "objects" in microscopic images, preferably in an automatic manner. Various labeled molecules (e.g., proteins or protein complexes), sub-cellular organelles, cells, or super-cellular objects (e.g., neuron populations or cell lineages) often need to be extracted, named, and compared with each other, before they can be measured. Most microscopic image analysis techniques can be categorized into three major classes, namely segmentation, registration, and annotation.

- Segmentation is the process of partitioning an image into multiple regions, so that voxels within each region share certain common features. Image segmentation is often used to locate objects and their boundaries (lines, curves, etc., e.g., [23]), as well as to

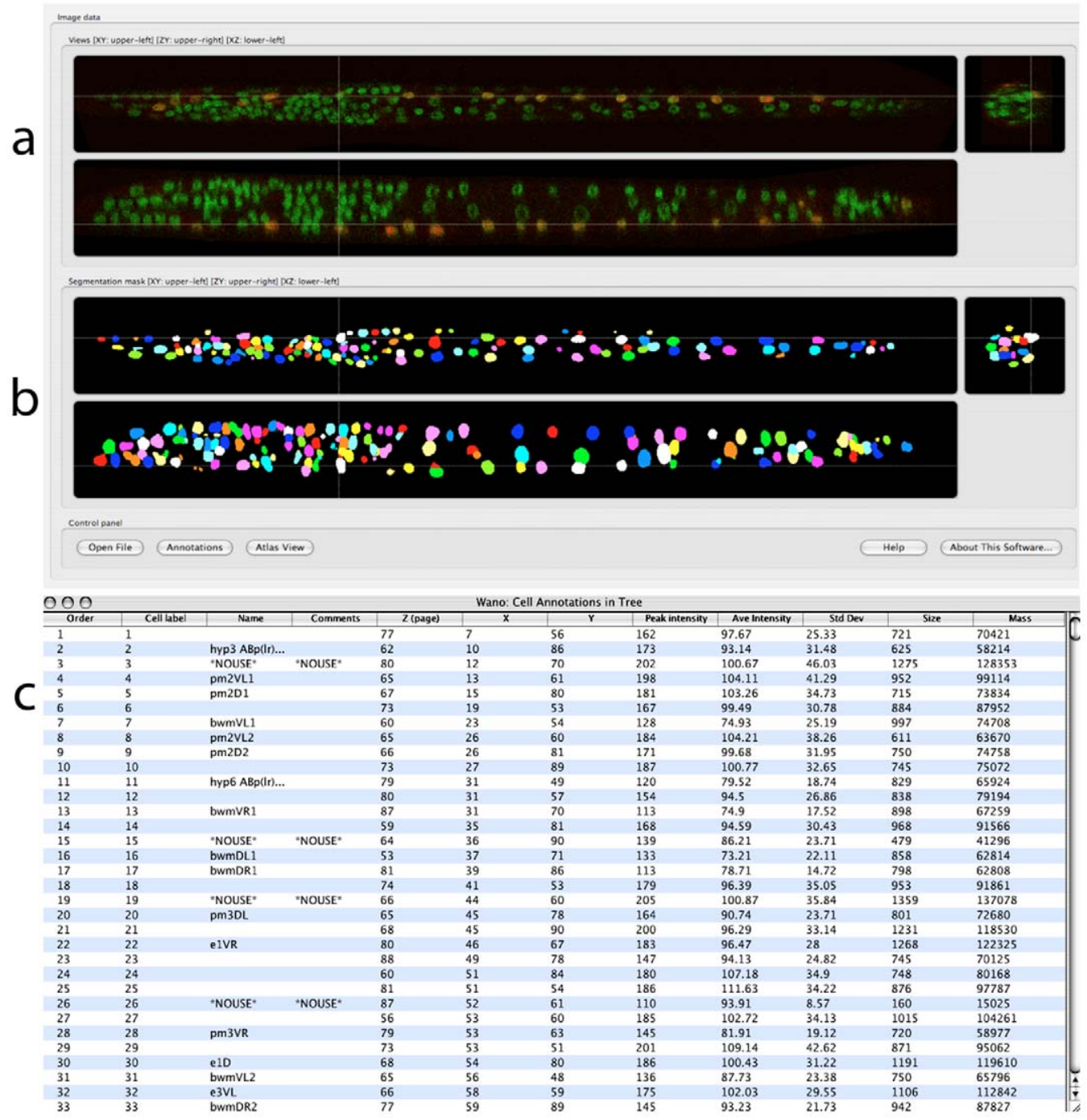

Figure 3. 3D image visualization and analysis for measuring single-cell gene expression of $C$. elegans. (a) Tri-view display of a confocal image of C. elegans (L1 stage). Green: DAPI staining (pseudo-colored); red: myo3:GFP labeled muscle cells. (b) Tri-view display of the 3D watershed segmented nuclei of (a). The co-localized image objects are indicated by crosses (white). (c) A spreadsheet display of 3D measured gene expression of various cells. All sub-figures are produced using VANO [39], a 3D annotation tool.

doi:10.1371/journal.pcbi.1002519.g003 
perform qualitative and quantitative analysis in images [24,25]. In microscopic image analysis, segmentation is typically used to locate, track, and classify bio-structures such as cells or nuclei [4], fibrous structures (e.g., axonal fibers [5,7], microtubules [26]), and anatomical/functional tissue regions. Thresholding [27], watershed $[28,25]$, and deformable models [29] are the basis for the most commonly used segmentation techniques for microscopic images.
- Registration [30] is the process to map multiple or many images geometrically, via a linear or nonlinear transform, so that image objects or features can be compared directly in a "standard" space. Registration is particularly widely used in three types of microscopic image processing tasks: stitching of image tiles [31] (e.g., electron and light microscopic tiles), registration of multiple samples of the same biological entity [19,32] (e.g., different images of the same neuron-population), fusion of multi-different views [33] of one object (e.g., tomography used in electron microscopy or selective plane illumination microscopy). Rigid or affine transforms are often used to register images globally. These linear transforms can be iteratively applied to images at different scales to achieve nonlinear registration. However, it is more common to use B-spline or thinplate-spline to derive nonlinear smooth transforms [34], which are often used to register images locally.
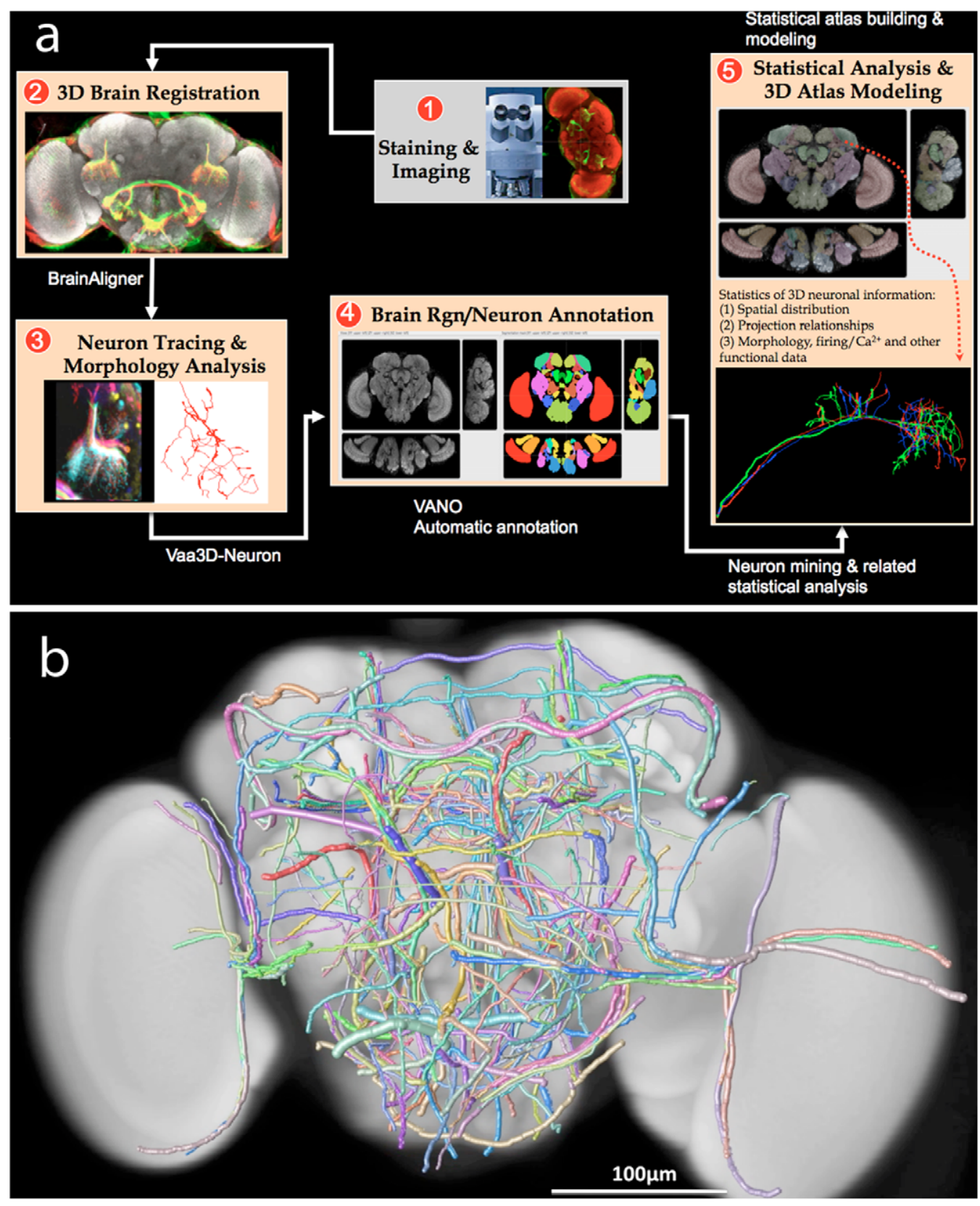

Figure 4. A pipeline of image analysis and data mining tools for building the neuronal atlases of fruit fly brains. (a) A flowchart of the key steps in building a fruit fly brain atlas. (b) A 3D digital atlas of 269 stereotyped neurite tracts reconstructed from GAL4-label fruit fly brains [19]. Pseudo colors are used to distinguish different tracts. The width of each tract equals its spatial divergence.

doi:10.1371/journal.pcbi.1002519.g004 
- Annotation is the process to label/ name images or image objects (e.g., cells) or assign their phenotypic properties with predefined terms. For example, controlled vocabularies of ontology have been assigned to images for annotating gene expression patterns (e.g., [35-37]). Another significant type of application is to recognize special objects of interest (e.g., cells) automatically $[4,38]$ and therefore to facilitate the quantitative measurement of biological entities (e.g., single-cell resolution gene expression).

\section{Pipelining 3D Visualization and Analysis Modules}

In many biological applications, different image analysis techniques need to be used as a whole pipeline. For instance, for profiling the gene expression at the single nucleus resolution of Caenorhabditis elegans [4], laser scanning microscopic images of this animal are first straightened (Figure 3a) [40], which can be categorized as a registration step. Then, $C$. elegans cells that are stained using DAPI are segmented (Figure 3b) using an adaptive 3D watershed algorithm. Cells are then recognized (Figure 3) based on their relative location

\section{References}

1. Inoué S (2008) Microtubule dynamics in cell division: exploring living cells with polarized light microscopy. Cell Develop Biol 24: 1-28.

2. Rübel O, Weber GH, Huang M-Y, Bethel EW, Biggin M, et al. (2010) Integrating data clustering and visualization for the analysis of $3 \mathrm{~d}$ gene expression data. IEEE Trans Comput Biol Bioinformatics 71: 64-79.

3. Rafalska-Metcalf IU, Janicki SM (2007) Show and tell: visualizing gene expression in living cells J?Cell Science 120(14): 2301-2307.

4. Long F, Peng H, Liu X, Kim SK, Myers E (2009) A 3D digital atlas of C. elegans and its application to single-cell analyses. Nat Methods 69: 667-672.

5. Peng H, Ruan Z, Atasoy D, Sternson S (2010) Automatic reconstruction of $3 \mathrm{~d}$ neuron structures using a graph-augmented deformable model. Bioinformatics 2612: 38-46.

6. Peng H, Long F, Myers E (2011) Automatic 3D neuron tracing using all-path pruning. Bioinformatics 2713: i239-i247.

7. Meijering E, Jacob M, Sarria JC, Steiner P, Hirling H, Unser M (2004) Design and validation of a tool for neurite tracing and analysis in fluorescence microscopy images. Cytometry 58A: 167-176.

8. Peng H (2008) Bioimage informatics: a new area of engineering biology. Bioinformatics 2417: 1827-1836.

9. Shamir L, Delaney JD, Orlov N, Eckley M, Goldberg IG (2010) Pattern recognition software and techniques for biological image analysis. PLoS Comput Biol611:e100097410.1371/journal.pcbi. 1000974.

10. Ljosa V, Carpenter AE (2009) Introduction to the quantitative analysis of two-dimensional fluorescence microscopy images for cell-based screening. PLoS Comput Biol 5(12): e1000603. 10.1371/ journal.pcbi. 1000603 . patterns in the 3D standardized space. Once the cell identities are determined, quantifying the gene expression is as simple as computing the normalized intensity within the nucleus region. The segmentation and recognition steps can also be unified using a recent approach of atlas-to-image deforming model [38].

Pipelining image analysis modules and other more sophisticated data analysis/ mining modules is a powerful way to generate quantitative biology. One such pipeline is shown in Figure 4a, which illustrates the main steps to construct the first 3D map of spatially invariant neurite tracts of a brain. Confocal images of adult fruit fly brains are first registered in 3D using the BrainAligner system [19] (Figure 4a, Step 2), so that different populations of neurons labeled using a number of GAL4 lines can be aligned and compared within the same 3D coordinate system. Then, neurite tracts are segmented and traced in 3D (Figure 4a, Step 3). The neurite tracts reconstructed from the same GAL4 line have a clear correspondence. They are then annotated (Figure 4a, Step 4). A neuron/neurite comparison and mining module is then used to determine the spatial divergence of the corresponding neurite tracts (Figure 4a, Step 5),

11. Helmstaedter M, Mitra PP (2012) Computational methods and challenges for large-scale circuit mapping. Curr Opin Neurobiol 22(1): 162-169.

12. Peng H, Long F, Zhao T, Myers EW (2011) Proof-editing is the bottleneck of $3 \mathrm{~d}$ neuron reconstruction: the problem and solutions. Neuroinformatics 92-3: 103-105.

13. Abramoff MD, Magelhaes PJ, Ram SJ (2004) Image processing with ImageJ. Biophotonics Int 11: 36-42.

14. Peng H, Ruan Z, Long F, Simpson JH, Myers EW (2010) V3D enables real-time 3D visualization and quantitative analysis of large-scale biological image data sets. Nat Biotechnol 284 348-353.

15. Gouaillard A, Brown T, Bronner-Fraser M, Fraser SE, Megason SG (2007) GoFigure and The Digital Fish Project: open tools and open data for an imaging based approach to system biology. Insight Journal - special edition "2007 MICCAI Open Science Workshop".

16. Cardona A, Saalfeld S, Preibisch S, Schmid B, Cheng A, et al. (2010) An integrated micro- and macroarchitectural analysis of the drosophila brain by computer-assisted serial section electron microscopy. PLoS Biol 810: e 1000502 doi:10.1371/journal.pbio. 1000502

17. Kitajima TS, Ohsugi M, Ellenberg J (2011) Complete kinetochore tracking reveals errorprone homologous chromosome biorientation in mammalian oocytes. Cell 146(4): 568-581.

18. Liu X, Long F, Peng H, Aerni SJ, Jiang M, et al (2009) Analysis of cell fate from single-cell gene expression profiles in C. elegans. Cell 139: 623633.

19. Peng H, Chung P, Long F, Qu L, Jenett A, et al. (2011) BrainAligner: 3D registration atlases of Drosophila brains. Nat Methods 8: 493-498. followed by a final mapping to the standard space of the 3D fly brain atlas (Figure 4a, Step 5). With this approach, it is possible to measure hundreds of stereotyped neurite tracts in a fruit fly's brain (Figure 4b). The same pipeline can be used to study other brain wiring-maps of neurons.

\section{Conclusions}

Visualization and analysis methods are critical for understanding and using 3D microscopic images for various cell biology, structural biology, neurosciences, and systems biology applications. These tools become indispensable for the ever-increasing need to screen tens of gigabytes to many terabytes of microscopic images. Pipelining these tools and other data analysis/mining methods is a new trend for producing interesting biology.

\section{Acknowledgments}

We thank Yang Yu, Ting Zhao, Hang Xiao, and Yinan Wan for discussion of this article, Jan Ellenberg, Philipp Keller, Rex Kerr, Stuart Kim, Tomoya Kitajima, Xiao Liu, Davi Bock, Clay Reid, and Julie Simpson for providing microscopic images, and Christine Morkunas for proofreading the manuscript.
20. Bock D, Lee W-C, Kerlin AM, Andermann ML, Yurgenson S, et al. (2011) Network anatomy and in vivo physiology of a group of visual cortical neurons. Nature 471: 177-182.

21. Keller P, Schmidt AD, Wittbrodt J, Stelzer EHK (2008) Reconstruction of zebrafish early embryonic development by scanned light sheet microscopy. Science 322(5904): 1065-1069.

22. Lorensen WE, Cline HE (1987) Marching Cubes: A high resolution 3D surface construction algorithm. Comput Graph 21(4): 163-169.

23. Baker ML, Yu Z, Chiu W, Bajaj C (2003) Automated segmentation of molecular subunits in electron cryomicroscopy density maps. J?Struct Biol 156(3): 432-441

24. Danuser G (2011) Computer vision in cell biology. Cell 147(5): 973-978.

25. Carpenter AE, Jones TR, Lamprecht MR, Clarke C, Kang IH, et al. (2006) CellProfiler: image analysis software for identifying and quantifying cell phenotypes. Genom Biol 7: R100.

26. Sargin ME, Altinok A, Kiris E, Feinstein SC, Wilson L, et al. (2007) Tracing microtubules in live cell images. ISBI 2007. pp. 296-299.

27. Sieracki ME, Reichenbach SE, Webb KL (1989) Evaluation of automated threshold selection methods for accurately sizing microscopic fluorescent cells by image analysis. Appl Environ Microbiol 55(11): 2762-2772.

28. Vincent L, Soille P (1991) Watersheds in digital spaces: an efficient algorithm based on immersion simulations. IEEE Trans Pattern Anal Machine Intell 13(6): 583-598.

29. McInerney T, Terzopoulos D (1996) Deformable models in medical image analysis: a survey. Med Image Anal 1(2): 91-108.

30. Zitová B, Flusser J (2003) Image registration methods: a survey. Imag Vis Comput 21(11): 977-1000. 
31. Yu Y, Peng H (2011) Automated high speed stitching of large 3D microscopic images. Proc. of IEEE 2011 Int. Symp Biomed Imaging: From Nano to Macro (ISBI'2011). pp. 238-241.

32. Jefferis GS, Potter CJ, Chan AM, Marin EC, Rohlfing T, et al. (2007) Comprehensive maps of Drosophila higher olfactory centers: spatially segregated fruit and pheromone representation. Cell 128: 1187-1203.

33. Rubio-Guivernau JL, Gurchenkov V, Luengo-Oroz MA, Duloquin L, Bourgine P, et al. (2012) Waveletbased image fusion in multi-view three-dimensional microscopy. Bioinformatics 28(2): 238-245.
34. Bookstein FL (1989) Principal warps: thin plate splines and the decomposition of deformations. IEEE Trans Pattern Anal Machine Intell 11: 567-585.

35. Zhou J, Peng H (2007) Automatic recognition and annotation of gene expression patterns of fly embryos. Bioinformatics 23(5): 589-596.

36. Peng H, Long F, Zhou J, Leung G, Eisen M, et al (2007) Automatic image analysis for gene expression patterns of fly embryos. BMC Cell Biol (Suppl 1): S7.

37. Li YX, Ji S, Kumar S, Ye J, Zhou Z-H (2010) Drosophila gene expression pattern annotation through multi-instance multi-label learning. IEEE/ACM Trans Comput Biol Bioinformatics 2009: 1445-1450.

38. Qu L, Long F, Liu X, Kim S, Myers E, et al. (2011) Simultaneous recognition and segmentation of cells: application in C. elegans. Bioinformatics 27(20): 2895-2902.

39. Peng H, Long F, Myers E (2009) VANO: a volume-object image annotation system. Bioinformatics 25(5): 695-697.

40. Peng H, Long F, Liu X, Kim S K, Myers EW (2008) Straightening Caenorhabditis elegans images. Bioinformatics 24(2): 234-242. 